\title{
Organizational challenges in the management of point-of-care diagnostics in healthcare facilities
}

https://doi.org/10.1515/labmed-2020-0008

Received January 24, 2020; accepted January 28, 2020; previously published online March 12, 2020

\begin{abstract}
This text is a synopsis of the "Seminar on Organizational Aspects of POCT Management” which was part of the $4^{\text {th }}$ Munich POCT symposium 2019. The session was chaired in part 1 by Christiane Maschek (Berlin) and Anke Urban (Ludwigshafen), and in part 2 by Barbara OschwaldHäg (Offenburg) and Marco Kachler (Klagenfurt/AT). The seminar was held in German in order to allow non-English-speaking medical technicians the full understanding of the presented contents. Part 1: Nice to Know - Challenges for networking POCT systems (Michaela Markhoff, Hamburg). Best Practice - Implementation of POCT in a hospital without central lab services (Jennifer Planz, Essen). Best Practice - Benefits of the POCT commission demonstrated using the example of implementing glucose POCT devices (Barbara Oschwald-Häg, Offenburg). Part 2: Best Practice - Challenges of a group-wide implementation of a POCT competence management (Sandra Mütze, Berlin). Nice to Know - Learning from mistakes in preanalytics (Reno Konzack, Berlin). New IFCC recommendation for checking reference intervals (Harald Maier, Altötting).
\end{abstract}

Keywords: best practice of POCT; POCT; POCT devices.

\section{Introduction}

This text is a synopsis of the "Seminar on Organizational Aspects of POCT Management" which was part of the $4^{\text {th }}$ Munich POCT symposium 2019. The session was chaired in part 1 by Christiane Maschek (Berlin) and Anke Urban (Ludwigshafen), and in part 2 by Barbara Oschwald-Häg

\footnotetext{
*Corresponding authors: Marco Kachler, Carinthia University of Applied Sciences and Deutsches Institut zur Weiterbildung für Technologen/-innen und Analytiker/-innen in der Medizin e.V. (DIW-MTA), Welserstr. 5-7, 10777 Berlin, Germany,

E-Mail:m.kachler@fh-kaernten.at; and

Christiane Maschek, Hannover Medical School and Dachverband für Technologen/-innen und Analytiker/-innen in der Medizin Deutschland e.V. (DVTA), Spaldingstr. 110b, 20097 Hamburg, Germany, E-Mail: maschek.christiane@mh-hannover.de
}

(Offenburg) and Marco Kachler (Klagenfurt/AT). The seminar was held in German in order to allow non-English speaking medical technicians the full understanding of the presented contents.

Part 1:

- Nice to Know - Challenges for networking POCT systems (Michaela Markhoff, Hamburg).

- Best Practice - Implementation of POCT in a hospital without central lab services (Jennifer Planz, Essen).

- Best Practice - Benefits of the POCT commission demonstrated using the example of implementing glucose POCT devices (Barbara Oschwald-Häg, Offenburg).

Part 2:

- Best Practice - Challenges of a group-wide implementation of a POCT competence management (Sandra Mütze, Berlin).

- Nice to Know - Learning from mistakes in preanalytics (Reno Konzack, Berlin).

- New IFCC recommendation for checking reference intervals (Harald Maier, Altötting).

\section{Sophisticated implementation of POCT in healthcare settings}

The miniaturization of analytical instruments has sparked an inexorable trend in laboratory diagnostics, which is becoming increasingly important as a point-of-care testing (POCT). This technological development is paired with the desire of clinicians to quickly obtain laboratory results at all times by providing appropriate POCT systems. An organizational challenge in Germany is the proper implementation of POCT in the clinical setting, compliant with the Guideline of the German Medical Association on Quality Assurance in Medical Laboratory Examinations (Rili-BÄK). The introduction of these POCT systems is a highly complex matter of great medical and economic significance and it makes sense to illuminate this complex topic from several perspectives (patients, organization, biomedical analytics). 
From the patient's point of view the advantages of POCT are obvious, the laboratory result is quickly available and a medical decision can be made immediately.

However, ensuring patient safety with POCT is a major challenge. Above all, the identification of patients must be organized in a way that is completely safe from confusion, in addition to the comprehensible documentation of the examination results. In many places, patient identification tapes are in place making a clear identification of patients possible. Missing or untraceable documentation during the archiving period is unacceptable. Necessarily, systems with online transfer of the analysis results to the laboratory information system (LIS) or hospital information system (HIS) have to be introduced and maintained.

From the point of view of the organization of the individual healthcare setting, this means that POCT must be implemented intelligently in order to minimize friction losses, ensure success (outcome) and at the same time understand and protect the various interests from the point of view of the individual factors.

To ensure that a certain POCT can actually improve the quality of patient care, proper examination has to take place beforehand. One problem is still the poorer precision and accuracy of the measurement results achieved by POCT systems in direct comparison with centralized laboratory diagnostics [1, 2]. The quality of the POCT must be ensured, i.e. in the practical consequence also a RiliBÄK-compliant implementation [3] of a quality assurance strategy. An interdisciplinary project team is an essential key factor for successful implementation and one of the prerequisites for sustainable and thus successful quality and data management. The POCT commission under the leadership of a POCT manager is the decisive steering committee, the driving force behind the introduction, implementation and further development of patient-oriented laboratory diagnostics. In the POCT commission, all persons and interfaces involved in the process have to be considered. The different questions and tasks can be discussed and assigned in this committee.

\section{Special view of the biomedical analyst}

From the point of view of the biomedical analysts, who as POCT managers have to organize and control the process, a central role has to be played. He/she is responsible for the administration of the POCT (measurement variables, devices and their IT networking, quality, data and document management including the quality control program according to the specifications of the Rili-BÄK or the ISO 22870 standard), the definition of maintenance measures and the organization of consumables.

In addition, a comprehensive training program for primary qualification and periodic re-qualification (competence management) of the POCT officers and users must be developed, implemented and documented. In this context it is important to mention that two poster award winners also examined this topic - Thomas Hug, Berlin: Establishing of a new POCT training concept for care-givers, and Rolf Bikker, Hannover: Development and integration of operator-optimized POCT eLearning modules.

A risk management system must be set up for prevention and error correction, because today the available assessment instruments (quality and plausibility checks) at POCT only record inaccurate pre-analytical factors of the effect on the analytical process. For example, internal quality controls only record the methodological reliability of the POCT process, i.e. whether the analytical system can produce correct analytical values at all. However, patient preparation and sampling errors as well as patient-related influencing and disturbing variables are insufficiently recognized, as no specific assessment of the prepared test samples takes place. As the core competence of POCT users is usually in a different area and therefore compliance for quality assurance measures is rather low, the training courses must clearly emphasize the importance of quality assurance, also in order to guarantee patient safety.

Another problem is the comparability of the examination procedures. An essential task in this context is the verification of reference intervals for the POCT measurement methods [4]. A practicable and meanwhile widely accepted solution is the verification by means of indirect methods [5], i.e. a determination or verification using statistical analyses from routine data. For POC tests, on the other hand, experience is still poor. First investigations show that the new "indirect" methods are in principle also applicable for POCT. However due to different sensitivities of the methods, the measurement principles used and the widely differing patient collectives as well as their particularities are to be taken into account.

POCT therefore means more than just the simple handling of a laboratory device for immediate diagnostics, it also requires critical handling of the results. The plausibility check is of great importance and, despite the simple handling of the systems, requires profound user training of the clinical staff (as an important prerequisite) for sufficiently high analytical quality of the POCT. The important time advantage of POCT can be used effectively and efficiently only through comprehensive clinical management 
to improve medical and economic outcomes. A steering group in the form of a POCT commission under the leadership of a POCT manager is a sufficient and necessary structural element for the implementation of quality requirements in POCT.

Author contributions: All the authors have accepted responsibility for the entire content of this submitted manuscript and approved submission.

Research funding: None declared.

Employment or leadership: None declared.

Honorarium: None declared.

Competing interests: The funding organization(s) played no role in the study design; in the collection, analysis, and interpretation of data; in the writing of the report; or in the decision to submit the report for publication.

\section{References}

1. Christensen TD, Larsen TB. Precision and accuracy of pointof-care testing coagulometers used for self-testing and selfmanagement of oral anticoagulation therapy. J Thromb Haemost 2012;10:251-60.

2. Dolscheid-Pommerich RC, Dolscheid S, Grigutsch D, StoffelWagner B, Graeff I. Comparability of point-of-care versus central laboratory hemoglobin determination in emergency patients at a supra-maximal care hospital. PLoS One 2016;11:e0166521.

3. Bundesärztekammer. Neufassung der Richtlinie der Bundesärztekammer zur Qualitätssicherung laboratoriumsmedizinischer Untersuchungen - Rili-BÄK. Dtsch Arztebl 2019;116:A-2422/B1990/C-1930.

4. Aufenanger J. Ein gordischer Knoten. Trillium Diagnostik 2018;16:122.

5. Jones GR, Haeckel R, Loh TP, Sikaris K, Streichert T, Katayev A, et al. Indirect methods for reference interval determination - review and recommendations. Clin Chem Lab Med. 2018;57:20-9. 